\title{
First-principles calculation of the graphene Dirac band on semi-infinite $\operatorname{Ir}(111)$
}

\author{
H. Ishida $\odot,{ }^{1}$ R. Arafune $\odot,,^{2}$ and N. Takagi $\odot{ }^{3}$ \\ ${ }^{1}$ College of Humanities and Sciences, Nihon University, Tokyo 156-8550, Japan \\ ${ }^{2}$ International Center for Materials Nanoarchitectonics, National Institute for Materials Science, \\ 1-1 Namiki, Tsukuba, Ibaraki 304-0044, Japan \\ ${ }^{3}$ Graduate School of Human and Environmental Studies, Kyoto University, Kyoto, Japan
}

(Received 8 September 2020; revised 6 November 2020; accepted 9 November 2020; published 20 November 2020)

\begin{abstract}
We study the energy dispersion relation of the $\pi$ and $\pi^{*}$ bands in epitaxial monolayer graphene on a semi-infinite $\operatorname{Ir}(111)$ substrate by a first-principles density-functional calculation. For this purpose, we employ a realistic surface structure in which the $(10 \times 10)$ unit cell of graphene matches a $(9 \times 9)$ cell of $\operatorname{Ir}(111)$. We determine the surface geometry by using a slab model containing four Ir layers, and the optimized structure is used as input for the subsequent surface embedded Green's function calculation. By taking advantage of semi-infinite calculations, we discuss mini energy gaps at the crossing of the $\pi$ band and its replicas, the Rashba-type spin splitting of the $\pi$ and $\pi^{*}$ bands, and also the energy width of both bands arising from interactions with the energy continuum of bulk Ir bands.
\end{abstract}

DOI: 10.1103/PhysRevB.102.195425

\section{INTRODUCTION}

Epitaxially grown graphene on metal substrates has been a subject of intense study in the fields of basic science and engineering over the last decade [1-7]. One of the central questions here concerns to what extent the $\pi$ and $\pi^{*}$ bands of freestanding graphene are modified due to symmetry lowering and orbital hybridizations upon adsorption on a metal substrate. The reason is that the unique transport properties of freestanding graphene arise from linear energy dispersion of the two bands with two-dimensional (2D) wave vector $\mathbf{k}$ in the vicinity of the $K$ and $K^{\prime}$ points of the Brillouin zone, which can be expressed via massless Dirac fermions as

$$
\varepsilon(\mathbf{k})=E_{D} \pm \hbar v_{F}\left|\mathbf{k}-\mathbf{k}_{D}\right|
$$

where $v_{F}$ is the Fermi velocity, $\mathbf{k}_{D}$ is the wave vector at the $K$ or $K^{\prime}$ point, and $E_{D}$ denotes the Dirac point energy [8]. Broadly speaking, the graphene/metal interfaces can be divided into two categories depending on the nature of graphene-substrate bonding. In the case of strong bonding (chemisorption), the graphene $\pi$ states are heavily intermixed with substrate $d$ bands, leading to massive modifications of the linear energy dispersion. In the case of weak bonding (physisorption), the $\pi$ states interact only weakly with substrate $s p$ bands, so that their linear energy dispersion is mostly preserved, except that a small gap $E_{g}$ can be opened between the $\pi$ and $\pi^{*}$ bands at $E_{D}[1,2,5]$.

Published by the American Physical Society under the terms of the Creative Commons Attribution 4.0 International license. Further distribution of this work must maintain attribution to the author(s) and the published article's title, journal citation, and DOI.
In the present work, we focus on monolayer graphene on $\operatorname{Ir}(111)$, which has been regarded as an example of relatively weakly interacting overlayers despite the existence of Ir $5 d$ states near the Fermi level $E_{F}$. Since the lattice constant of graphene is $10 \%$ smaller than that of the $\operatorname{Ir}(111)$ plane, superposition of the two lattices gives rise to moiré superstructures [9-13], which depends on the relative rotational angle $\phi$ of the two lattices. It was revealed that $\phi$ can be controlled by changing the temperature $T$ of the $\mathrm{Ir}$ substrate during graphene growth. At high temperatures $(T \geqslant$ $1500 \mathrm{~K}$ ), the perfectly aligned structure with $\phi=0^{\circ}$ (termed $R 0^{\circ}$ phase) is formed, whereas at lower temperatures, several distinct moiré structures with finite rotational angles were found $[14,15]$. The $R 0^{\circ}$ overlayer can be described accurately by a commensurate structure where a $(10 \times 10)$ supercell of graphene matches a $(9 \times 9)$ cell of $\operatorname{Ir}(111)$. Within the supercell, the overlayer exhibits undulations perpendicular to the surface [11-13].

The occupied part of the electronic structure of the $R 0^{\circ}$ graphene/ $\operatorname{Ir}(111)$ was thoroughly investigated by angleresolved photoemission spectroscopy (ARPES) [15-27]. The main findings are as follows: (i) Overall, the linear energy dispersion of the $\pi$ band is preserved. (ii) The Dirac point estimated from the fitting of the linear dispersion of the $\pi$ band is located slightly above $E_{F}$, implying small charge transfer to the substrate $[16,18,20]$. (iii) The summit of the $\pi$ band, $E_{\pi}=E_{D}-E_{g} / 2$, is either slightly above $E_{F}$ [16] or below $E_{F}[19,20]$. (iv) The moiré potential produces replicas of the $\pi$ bands centered at $\mathbf{k}=\mathbf{k}_{D}+\mathbf{G}$ with $\mathbf{G}$, a reciprocal lattice vector corresponding to the superlattice. (v) Mini energy gaps are observed at the crossing of the primary and replica $\pi$ bands [16] and also at the crossing of the $\pi$ band and $\mathrm{Ir}$ surface bands [18]. (vi) Hybridizations between the $\pi$ and $\mathrm{Ir}$ $5 d$ states give rise to the spin-orbit splitting of the $\pi$ band of $\sim 50 \mathrm{meV}$ [15]. (vii) The Dirac point can be shifted to lower 
energies below $E_{F}$ via electron doping through adsorption or intercalation of chemical species [18-26].

On the theory side, several density-functional theory (DFT) calculations were reported for the $(10 \times 10)$ graphene/Ir(111) surface and the related ones with additional adsorbates or intercalated species $[1,9,11,26-31]$. These calculations employed a slab model containing about five $\operatorname{Ir}(111)$ layers to represent the substrate. For the purpose of structural optimization, the use of a thin slab is justified since the electron density at the surface converges very rapidly with increasing slab thickness for metal substrates with a high bulk electron density such as Ir. On the other hand, upon adsorption, the electronic states in the overlayer interact with the energy continuum of the substrate and become resonances with a finite energy width unless they are located within a projected bulk band gap of the substrate. However, if the substrate is modeled by a thin slab, all energy levels in the surface normal direction are discretized. Thus, the slab model cannot correctly describe the interaction between the overlayer and substrate electronic states. The unfolded band structure of the $(10 \times 10)$ graphene overlayer on $\operatorname{Ir}(111)$, including the spin-orbit splitting and energy gaps, was presented in a few recent papers $[1,29,30]$. Since the Ir substrate in those works was represented by an aproximately five-layer-thick slab, it is important for comparison with realistic surfaces to examine how the band structure converges with increasing substrate thickness. As such, in the present work, we calculate the electronic structure of graphene monolayer on a truly semi-infinite substrate. To do so, we employ a realistic $(10 \times 10)$ graphene overlayer on a $(9 \times 9)$ $\operatorname{Ir}(111)$ substrate. We discuss not only the energy dispersion of the $\pi$ and $\pi^{*}$ bands but also their energy widths as a function of $\mathbf{k}$.

The outline of this paper is as follows. In Sec. II we present the surface geometry of the $R 0^{\circ}$ graphene on $\operatorname{Ir}(111)$, which will be used for the subsequent electronic structure calculation. Section III contains the results and discussion of the present semi-infinite surface calculations. We begin with the discussion of the electronic structure of the clean $\operatorname{Ir}(111)$ and three hypothetical $1 \times 1$ graphene overlayers in which their lattice constant is artificially enlarged to match that of the $\operatorname{Ir}(111)$ substrate. Then, we proceed to provide core results of the present paper, i.e., those for the realistic $(10 \times 10)$ graphene overlayer on a $(9 \times 9) \operatorname{Ir}(111)$ substrate. We conclude in Sec. IV.

\section{SURFACE GEOMETRY}

We choose the [110], [1̄1 2$]$, and [111] crystal orientations of fcc Ir as the positive $x, y$, and $z$ directions, respectively. The surface geometry of the $R 0^{\circ}$ graphene monolayer forming a $(10 \times 10)$ structure on a $(9 \times 9)$ supercell of $\operatorname{Ir}(111)$ is determined by total-energy minimization within DFT. It is known that the generalized gradient approximation (GGA) in DFT substantially overestimates the interlayer spacing between graphene and the $\operatorname{Ir}(111)$ substrate, since the bonding between them arises from the van der Waals ( $\mathrm{vdW}$ ) interaction which is absent from the GGA [9]. Recently, much effort has been made to develop a theoretical framework called vdW-DFT, where the nonlocal correlation energy functional accounting for the vdW interaction is added to the GGA
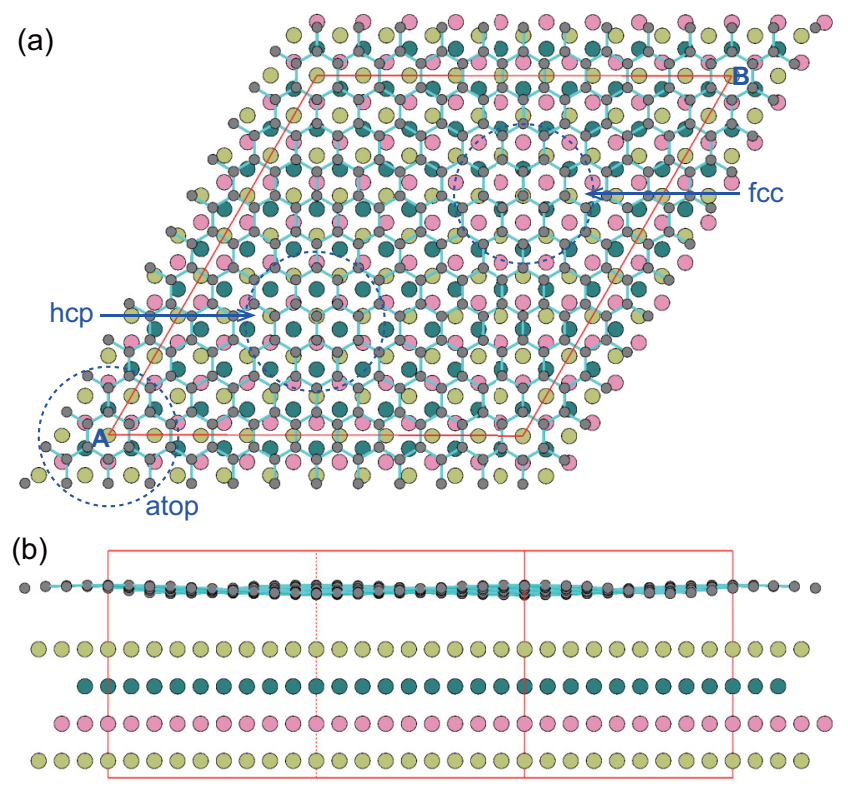

FIG. 1. (a) Top and (b) side views of the $(10 \times 10)$ graphene monolayer on a $(9 \times 9) \operatorname{Ir}(111)$ slab containing four Ir layers. The red parallelogram indicates a $(9 \times 9)$ supercell of the substrate. Three dashed circles encompass regions where carbon hexagons are located at atop, hcp, and fcc adsorption sites.

functional $[32,33]$. The vdW-DFT was able to predict the experimentally observed atomic structure of graphene on $\operatorname{Ir}(111)$ precisely [11-13]. Though we fully acknowledge this fact, in the present work we have employed the local density approximation (LDA) in DFT for the following reasons: (i) Feibelman demonstrated that LDA can reproduce the atomic structure of the graphene overlayer on $\operatorname{Ir}(111)$ excellently, although this agreement may be of fortuitous nature due to error cancellations [31,32]. (ii) We need the optimized geometry as input for the subsequent surface Green's function calculation and do not aim to discuss bonding energies of the overlayer. (iii) LDA can often predict the work function of metals better than GGA. In the case of the clean $\operatorname{Ir}(111)$ surface, the experimental value is $5.79 \mathrm{eV}$ [34], while the LDA and GGA values are 5.86 and $5.51 \mathrm{eV}$, respectively, according to Ref. [35], which agree well with 5.88 and $5.51 \mathrm{eV}$ obtained with the present Green's function calculation. The difference in work function between LDA and GGA may affect the energy levels relative to $E_{F}$ of weakly bound electrons.

The calculation is made with the use of the VASP program [36,37], an implementation of the projector augmented-wave method [38]. The surface is modeled by a slab model that consists of a graphene monolayer and four $\operatorname{Ir}(111)$ layers. All atoms except for those in the bottom Ir layer are allowed to relax. The lattice constant of the hexagonal $\operatorname{Ir}(111)$ plane, $a_{\text {Ir }}=2.71 \AA$ was determined by a separate bulk total-energy calculation.

In Fig. 1 we show the top and side views of the surface geometry of the $(10 \times 10)$ graphene overlayer on a $(9 \times 9) \operatorname{Ir}(111)$ slab obtained by the present calculation. The averaged interlayer spacing between the overlayer and the outermost Ir layer, $3.40 \AA$, is in good agreement with previous 
measurements [11-13] and with the vdW-DFT calculation [11]. The three dashed circles in Fig. 1(a) labeled atop, hcp, and fcc encompass distinct regions in a supercell where the center of a carbon hexagon is located approximately on top of an Ir atom in the outermost, the second, and the third Ir layer, respectively. The calculated heights relative to the outermost Ir layer are $3.73,3.16$, and $3.18 \AA$ for the atop, fcc, and hcp adsorption sites, respectively, implying that the undulation of the overlayer in the normal $(z)$ direction amounts to $0.57 \AA$. This value is by $0.1 \AA$ larger than the vdW-DFT value, $0.46 \AA$ [11]. The perpendicular undulation within the substrate layers, which decays with distance from the surface, is much smaller. Its magnitude for the outermost Ir layer is $\sim 0.01 \AA$.

\section{SURFACE ELECTRONIC STRUCTURE}

We employ the embedded Green's function (EGF) technique of Inglesfield $[39,40]$ to calculate the electronic structure of semi-infinite surfaces. In this method, the space is partitioned into the surface region with finite thickness and the semi-infinite substrate, and the Green's function in the embedded surface region is computed by representing the semi-infinite bulk via the embedding potential acting on the boundary surface between the two regions. We use a computer code $[41,42]$ that combines the EGF technique and the full-potential linearized augmented plane-wave (LAPW) method [43]. The spin-orbit interaction inside the muffin-tin sphere surrounding each nucleus is incorporated by adding the standard $\vec{l} \cdot \vec{\sigma}$ term to the embedded Hamiltonian, where $\vec{l}$ is the orbital angular momentum operator and $\vec{\sigma}=\left(\sigma_{x}, \sigma_{y}, \sigma_{z}\right)$ is the vector of Pauli matrices. To calculate the surface Green's function, we invert a double-sized matrix containing the Hamiltonian for both spin components without using any perturbation approach. To be consistent with the structural calculation, self-consistent surface electronic structure calculations are performed within the LDA in DFT.

\section{A. Clean $\operatorname{Ir}(111)$ surface}

First, we examine the surface band structure of the clean $\operatorname{Ir}(111)$ surface with which the graphene overlayer interacts. We calculate the electronic structure of the ideal $\operatorname{Ir}(111)$ by including three Ir layers in the surface embedded region. The plane-wave cutoff for the LAPW basis functions in the interstitial region is chosen as $E_{c}=3.5^{2}$ Ry while radial orbitals with angular momentum $l \leqslant l_{\max }=6$ are included in the basis functions inside muffin-tin spheres.

In Fig. 2(a) we show $\rho(\mathbf{k}, \varepsilon)$, k-resolved density of states (DOS) projected on a first-layer Ir atom, along the $\Gamma-K\left(k_{x}\right)$ direction for the interval, $0.7 k_{s} \leqslant k_{x} \leqslant 1.3 k_{s}$, where $\mathbf{k}_{s}=$ $\left(k_{s}, 0\right)$ with $k_{s}=\frac{4 \pi}{3 a_{\mathrm{II}}}\left(1.55 \AA^{-1}\right)$ is the $K$ point of $\operatorname{Ir}(111)$. Here, $\rho(\mathbf{k}, \varepsilon)$ of an atom whose nucleus is located at $\mathbf{r}_{n}$ is defined by

$$
\rho(\mathbf{k}, \varepsilon)=\frac{-1}{\pi} \int_{\left|\mathbf{r}-\mathbf{r}_{n}\right| \leqslant R} d \mathbf{r} \operatorname{Tr}[\operatorname{Im} \hat{G}(\mathbf{k}, \omega, \mathbf{r}, \mathbf{r})] .
$$

In Eq. (2), the volume integral is performed within a muffintin sphere with radius $R$ centered at $\mathbf{r}_{n}$ and the trace is taken over spin index of the $2 \times 2$ Green's function matrix $\hat{G}(\mathbf{k}, \omega, \mathbf{r}, \mathbf{r})$ with $2 \mathrm{D}$ wave vector $\mathbf{k}$, complex energy $\omega=$

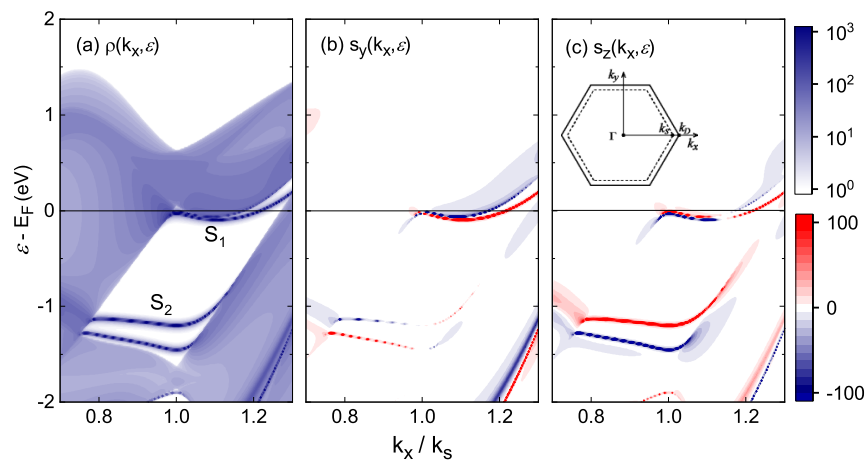

FIG. 2. Intensity plots of (a) $\rho(\mathbf{k}, \varepsilon)$, (b) $s_{y}(\mathbf{k}, \varepsilon)$, and (c) $s_{z}(\mathbf{k}, \varepsilon)$ calculated in a first-layer Ir muffin-tin sphere with radius $R=$ 2.48 bohrs for semi-infinite $\operatorname{Ir}(111)$ along the $k_{x}$ direction. The $K$ point of $\operatorname{Ir}(111)$ is located at $\mathbf{k}_{s}=\left(k_{s}, 0\right)$. Color varies linearly with $\log _{10}|\rho(\mathbf{k}, \epsilon)|$ in panel (a) and linearly with $\left|s_{\alpha}(\mathbf{k}, \epsilon)\right|$ in panels (b) and (c) (see color scale bars). The imaginary energy $\gamma=$ $2 \mathrm{meV}$. The inset in (c) illustrates the surface Brillouin zones of pristine $\operatorname{Ir}(111)$ (dashed hexagon) and free-standing graphene (solid hexagon).

$\varepsilon+i \gamma$, and space coordinate $\mathbf{r}$, where a small imaginary en$\operatorname{ergy} \gamma$ is introduced to broaden $\delta$-function-like DOS peaks of discrete surface bands.

In Figs. 2(b) and 2(c) we also show the $y$ and $z$ components of the k-resolved magnetization density projected on a firstlayer Ir atom defined by

$$
s_{\alpha}(\mathbf{k}, \varepsilon)=\frac{-1}{\pi} \int_{\left|\mathbf{r}-\mathbf{r}_{n}\right| \leqslant R} d \mathbf{r} \operatorname{Tr}\left[\sigma_{\alpha} \hat{G}(\mathbf{k}, \omega, \mathbf{r}, \mathbf{r})\right],
$$

where $\alpha=y$ or $z$. It should be noted that $s_{x}(\mathbf{k}, \varepsilon)$ vanishes identically on the $k_{x}$ axis due to the following reason: Let us denote the wave function of a nondegenerate surface state with a wave vector $\mathbf{k}=\left(k_{x}, 0\right)$ by $\psi_{s}$. We apply the time-reversal operator $\hat{T}$ and the mirror reflection operator with respect to the $y z$ plane, $\hat{M}$, to $\psi_{s}$. The resultant state $\hat{M} \hat{T} \psi_{s}$, which is also an energy eigenfunction due to the $C_{3 v}$ symmetry of the surface, should coincide with $\psi_{s}$ except for a constant $C$, i.e., $\hat{M} \hat{T} \psi_{s}=C \psi_{s}$ with $|C|=1$. Hence, the $x$ component of the spin operator has an expected value, $\left\langle\sigma_{x}\right\rangle=\left\langle\psi_{s}\left|\sigma_{x}\right| \psi_{s}\right\rangle=$ $\left\langle\hat{M} \hat{T} \psi_{s}\left|\sigma_{x}\right| \hat{M} \hat{T} \psi_{s}\right\rangle=-\left\langle\sigma_{x}\right\rangle$, indicating that $\left\langle\sigma_{x}\right\rangle=0$. Thus, the spin associated with $\psi_{s}$ is perpendicular to the $x$ axis. Furthermore, at $k_{x}=k_{s}$, the spin is perpendicular not only to the $x$ direction but also to the two symmetrically equivalent directions within the $x y$ plane (rotated by $\pm 120^{\circ}$ with respect to the $x$ axis), which implies that the spin must point to the surface normal $(z)$ direction.

It is seen that two pairs of spin-split surface bands, $S_{1}$ and $S_{2}$, emerge inside the surface projected bulk band gap having a distorted diamondlike shape. In the first Ir layer, $S_{2}$ is contributed dominantly by the $d_{y z}$ orbital. As is seen, $S_{2}$ exhibits a large spin-orbit splitting. The spin of the $S_{2}$ band is mainly polarized in the surface normal $( \pm z)$ direction. The $y$ component of the spin is several times smaller and changes sign at $k_{x}=k_{s}$ as discussed above. Seen from the positive $x$ direction, the spin rotates clockwise with increasing $k_{x}$. The orbital character of $S_{1}$ in the first Ir layer is dominantly $d_{3 z^{2}-r^{2}}$ like. $S_{1}$ exhibits a Rashba-type spin splitting whose magnitude 
increases with increasing $k_{x}$. The $y$ and $z$ components of the spin of $S_{1}$ are of similar size and the spin rotates anticlockwise with increasing $k_{x}$ when seen from the positive $x$ direction. The surface bands in Fig. 2 are in good agreement with the ARPES result of Pletikosić et al. [16], in which two spin-split branches of the present $S_{2}$ band were named $S_{2}$ and $S_{3}$.

\section{B. The (1×1) graphene overlayer on $\operatorname{Ir}(111)$}

Before moving on to the $(10 \times 10)$ graphene $/(9 \times 9)$ $\operatorname{Ir}(111)$ surface, we calculate the electronic structure of $(1 \times 1)$ flat graphene overlayers on $\operatorname{Ir}(111)$ to examine to what extent the local symmetry of different adsorption sites affects the energy dispersion of the graphene $\pi$ and $\pi^{*}$ bands. For this purpose, the planar lattice constant of graphene is increased artificially by $10 \%$ to match that of the $\operatorname{Ir}(111)$ plane, $a_{\text {Ir }}=2.71 \AA$. For the atop, hcp, and fcc geometries, we adopt the overlayer-substrate heights given in Sec. II. The cutoff parameters for the LAPW basis set are chosen as $E_{c}=3.5^{2}$ Ry and $l_{\max }=6$.

The first, second, and third rows of Fig. 3 display the calculated results for the atop, hcp, and fcc adsorption geometries, respectively. The first column of each row shows $\rho(\mathbf{k}, \varepsilon)$ averaged over two $\mathrm{C}$ atoms in a unit cell, while the second and the third show $\rho(\mathbf{k}, \varepsilon)$ and $s_{z}(\mathbf{k}, \varepsilon)$ of a first-layer Ir atom, respectively.

In freestanding graphene, the $\pi$ and $\pi^{*}$ bands are degenerate at the $K$ point since the two $\mathrm{C}$ atoms in a $(1 \times 1)$ unit cell (denoted as $C_{1}$ and $C_{2}$ sites) are symmetrically equivalent. In the case of the atop adsorption geometry, this symmetry is approximately preserved since the effects of the second- and third-layer Ir atoms located underneath the two $\mathrm{C}$ atoms are mostly screened out. As a result, the $\pi$ and $\pi^{*}$ bands shown in Fig. 3(a) exhibit no noticeable energy gap at $E_{D}$. Both the bands become surface resonances within the energy continuum of a projected bulk band. In Fig. 3(a), the degenerate DOS peak at $k_{x}=k_{s}$ is located at $0.18 \mathrm{eV}$ above $E_{F}$ and its full width at half maximum (FWHM) amounts to $25 \mathrm{meV}$. Figure 3(b) reveals that the $\pi$ band of graphene interacts with the surface bands of $\operatorname{Ir}(111), S_{1}$ and $S_{2}$, resulting in the opening of energy gaps at the crossing points. To demonstrate this more clearly, we show in the inset of panel (b) a magnified view of the DOS at the crossing point of the $\pi$ and $S_{1}$ bands. Strong distortion of the energy dispersion of the $\pi$ band due to the interaction with $S_{1}$ was observed in a previous ARPES experiment [18]. The hybridization of the $\pi$ band and Ir $5 d$ states results in spin splitting of the $\pi$ band. As seen from the inset of Fig. 3(c), which plots $s_{z}(\mathbf{k}, \varepsilon)$ of a first-layer Ir atom at $k_{x}=0.9 k_{s}$ within a small energy interval containing the $\pi$ band peaks, the spin-orbit splitting of the $\pi$ band amounts to $\sim 20 \mathrm{meV}$. Its magnitude changes only weakly with $k_{x}$.

In the case of the hcp and fcc adsorption geometries, the asymmetry of the two $\mathrm{C}$ atoms in a $(1 \times 1)$ unit cell is greatly increased, since first-layer Ir atoms are located, say, underneath the $C_{1}$ sites in the case of the hcp geometry and underneath the $C_{2}$ sites in the case of the fcc geometry. This results in strong modifications of the energy dispersion of the $\pi$ and $\pi^{*}$ bands as seen from Figs. 3(d) and 3(g). A large energy gap $(>0.5 \mathrm{eV})$ is opened between the $\pi$ and $S_{1}$ band. Further, the $\pi^{*}$ band, which is shifted downward and merged
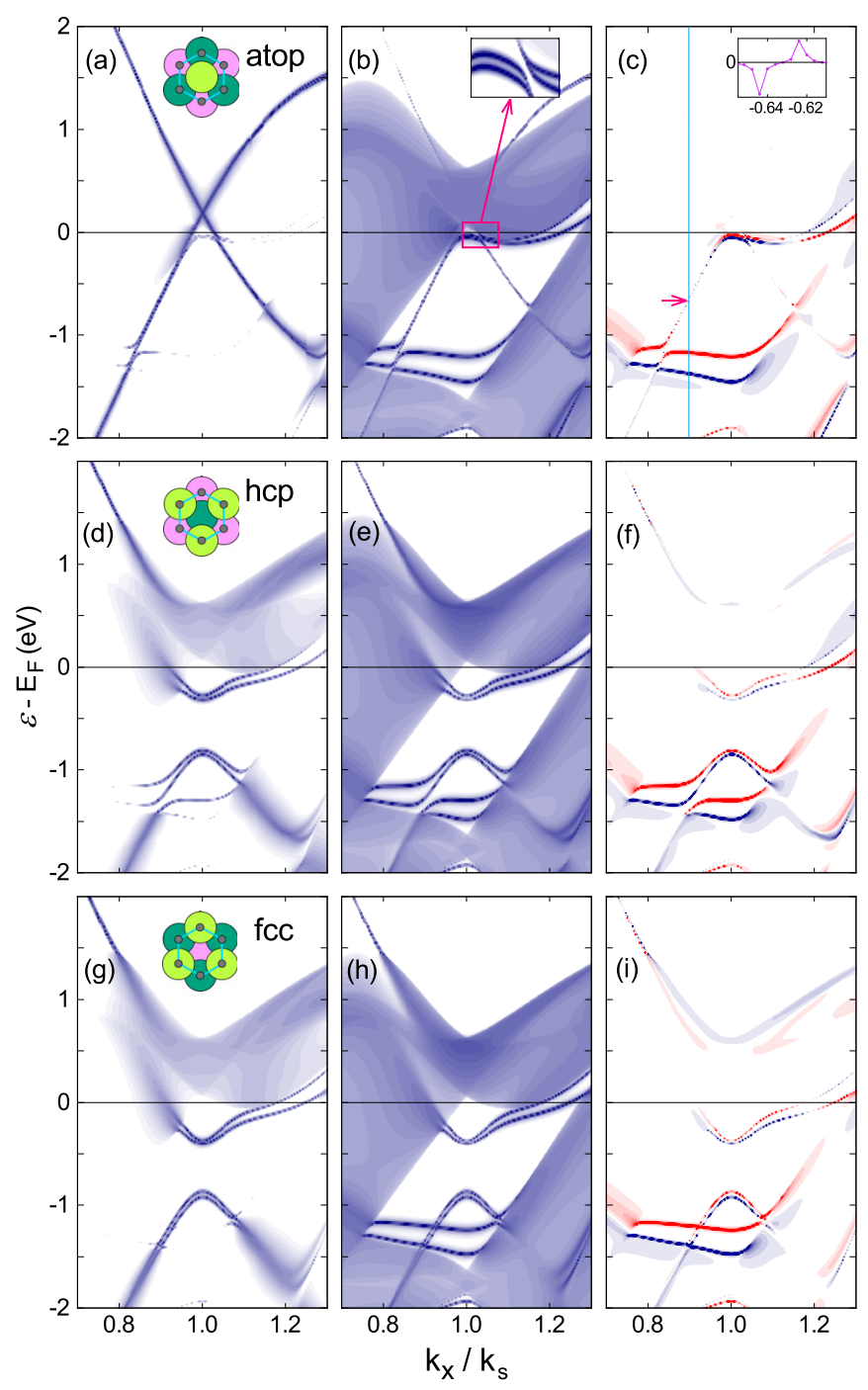

FIG. 3. (a), (d), (g) $\rho(\mathbf{k}, \varepsilon)$ averaged over two inequivalent $\mathbf{C}$ atoms. (b), (e), (h) $\rho(\mathbf{k}, \varepsilon)$ for a first-layer Ir atom. (c), (f), (i) $s_{z}(\mathbf{k}, \varepsilon)$ for a first-layer Ir atom. Panels (a)-(c), (d)-(f), and (g)-(i) correspond to the atop, hcp, and fcc adsorption geometries, respectively. The same color scales as those in Fig. 2 are used. Muffin-tin sphere radii are $R=1.33$ and 2.48 bohrs for $\mathrm{C}$ and $\mathrm{Ir}$ atoms, respectively. The imaginary energy $\gamma=2 \mathrm{meV}$. The inset in (b) displays a magnified view of the DOS at the band crossing. The inset in (c) displays $s_{z}(\mathbf{k}, \varepsilon)$ in a small energy interval containing two peaks of the spinsplit $\pi$ band marked by a red arrow.

within the projected bulk bands of Ir, is hardly seen except for a higher energy region with $k_{x}<0.8 k_{s}$.

\section{The $(10 \times 10)$ graphene overlayer on $\operatorname{Ir}(111)$}

Now, we calculate the electronic structure of graphene monolayer forming a $(10 \times 10)$ superstructure on a $(9 \times 9)$ cell of $\operatorname{Ir}(111)$ by adopting the surface geometry obtained in Sec. II. We include a graphene monloayer and the outermost Ir atomic layer in the surface embedded region, so that the embedded region contains $81 \mathrm{Ir}$ and $200 \mathrm{C}$ atoms per unit cell. It is an advantage of the EGF method that one needs to embed just a single substrate layer for accurate surface calculations in 

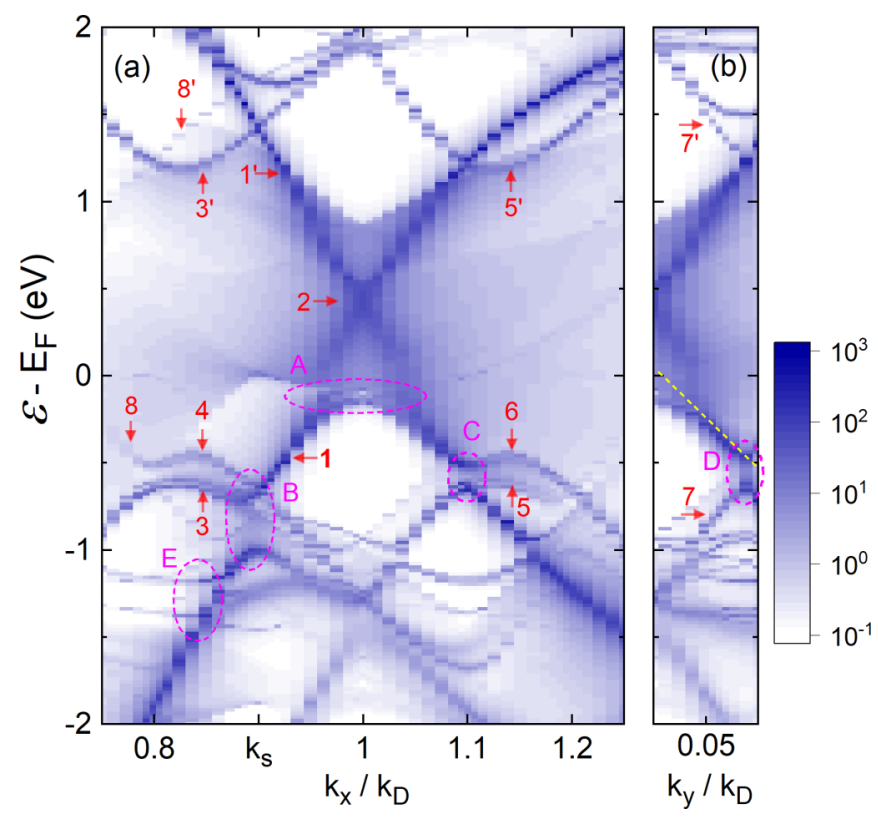

FIG. 4. Intensity plot of $\tilde{\rho}(\mathbf{k}, \varepsilon)$ for the $(10 \times 10)$ graphene overlayer on $\operatorname{Ir}(111)$. Color varies linearly with $\log |\tilde{\rho}(\mathbf{k}, \varepsilon)|$ (see color scale bar). (a) $\mathbf{k}=\left(k_{x}, 0\right)$ with $0.75 k_{D} \leqslant k_{x} \leqslant 1.25 k_{D}$ where $k_{x}=$ $k_{s}\left(=0.9 k_{D}\right)$ and $k_{x}=k_{D}$ correspond to the $K$ points of $\operatorname{Ir}(111)$ and graphene, respectively. (b) $\mathbf{k}=\left(k_{D}, k_{y}\right)$ with $0 \leqslant k_{y} \leqslant 0.1 k_{D}$. The muffin-tin sphere radius of $\mathrm{C}$ atoms is chosen as $R=1.33$ bohrs. $\Delta \varepsilon=20 \mathrm{meV}$ and $\gamma=2 \mathrm{meV}$.

the case of metals with high electron densities [44]. To reduce computational load without losing the numerical accuracy, we use $E_{c}=3.2^{2}$ Ry as a cutoff energy for the LAPW basis set, which is only slightly smaller than the one used in the preceding sections. With this $E_{c}$, the size of the Hamiltonian matrix to be inverted still amounts to $5.6 \times 10^{4}$. The Green's function is calculated in a small Brillouin zone (BZ) of the $(10 \times 10)$ graphene supercell. In order to obtain the energy dispersion of the $\pi$ and $\pi^{*}$ bands in the BZ of the $(1 \times 1)$ primitive cell, it is necessary to unfold the band structure. In the Appendix, we describe the method we used for unfolding the local DOS at a single $\mathbf{k}$ point in the supercell $\mathrm{BZ}$ onto those at $10^{2} \mathbf{k}$ points in the $\mathrm{BZ}$ of the primitive cell.

In Fig. 4 we show the intensity plot of $\tilde{\rho}(\mathbf{k}, \varepsilon)$, the kresolved DOS which is projected on the graphene overlayer and unfolded onto the $\mathrm{BZ}$ of the primitive cell. As described in the Appendix, we take the average of the local DOS over the $200 \mathrm{C}$ muffin-tin spheres within a supercell to calculate this quantity. Panel (a) shows $\tilde{\rho}(\mathbf{k}, \varepsilon)$ along the $k_{x}$ axis within the interval of $0.75 k_{D} \leqslant k_{x} \leqslant 1.25 k_{D}$, where $\mathbf{k}_{D}=\left(k_{D}, 0\right)$ with $k_{D}=\frac{10}{9} k_{s}\left(1.72 \AA^{-1}\right)$ the $K$ point of graphene. Panel (b) shows the local DOS in the perpendicular direction, i.e., $\tilde{\rho}(\mathbf{k}, \varepsilon)$ for $\mathbf{k}=\left(k_{D}, k_{y}\right)$ in the interval of $0 \leqslant k_{y} \leqslant 0.1 k_{D}$. We have employed different logarithmic color scaling than that in Figs. 2 and 3 to be able to illustrate weak DOS features arising from replica Dirac cones. Also, the DOS intensity maps in Fig. 4 exhibit more grainy features than those in Figs. 2 and 3 since we had to compromise on the density of $(\mathbf{k}, \varepsilon)$ mesh points due to the enormous computational time required for the $(10 \times 10)$ supercell calculation. In Fig. 4, the
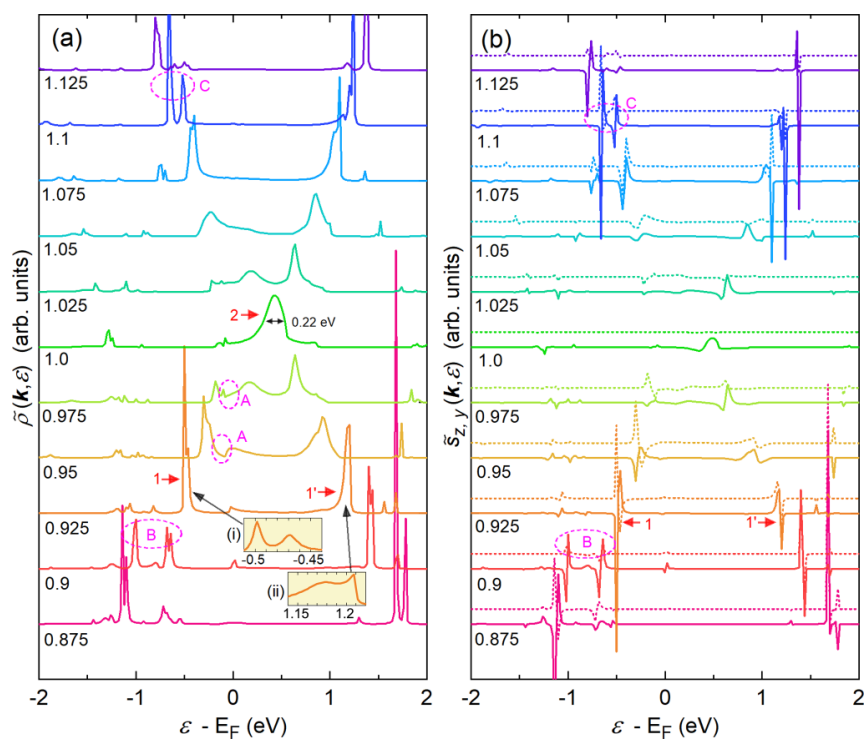

FIG. 5. (a) $\tilde{\rho}(\mathbf{k}, \varepsilon)$ of the $(10 \times 10)$ graphene overlayer on $\operatorname{Ir}(111)$ at $11 \mathbf{k}$ points on the $k_{x}$ axis. (b) $\tilde{s}_{y}(\mathbf{k}, \varepsilon)$ (dotted lines) and $\tilde{s}_{z}(\mathbf{k}, \varepsilon)$ (solid lines) at the same $k_{x}$ 's as in (a). $\Delta \varepsilon=20 \mathrm{meV}$ and $\gamma=2 \mathrm{meV}$ in both panels. The small number below each curve indicates $k_{x} / k_{D}$ where $\mathbf{k}_{D}=\left(k_{D}, 0\right)$ is the $K$ point of graphene. $\tilde{s}_{y}(\mathbf{k}, \varepsilon)$ and $\tilde{s}_{z}(\mathbf{k}, \varepsilon)$ at a given $k_{x}$ are vertically shifted for better visibility. The insets (i) and (ii) in panel (a) show the $\pi$ and $\pi^{*}$ band peaks at $k_{x}=0.925 k_{D}$ calculated with $\Delta \varepsilon=2 \mathrm{meV}$ and $\gamma=1 \mathrm{meV}$, respectively.

spacing between neighboring energy points $\Delta \varepsilon$ is $20 \mathrm{meV}$ and that between neighboring $\mathbf{k}$ points is partly $\Delta k=\frac{1}{160} k_{D}$ and partly $\frac{1}{80} k_{D}$, whereas the corresponding ones in Figs. 2 and 3 were $\Delta \varepsilon=4 \mathrm{meV}$ and $\Delta k=\frac{1}{600} k_{s}$.

First of all, by tracing two diagonal thick (dark-blue) lines gapped at a few places along the way in Fig. 4, we see that overall, the linear energy dispersion of the $\pi$ and $\pi^{*}$ bands is preserved. The dispersion curves of the two bands are qualitatively similar to the corresponding ones in Fig. 3(a) of the $(1 \times 1)$ overlayer except that the two bands cross at $k_{x}=k_{D}$ in the present case instead of at $k_{x}=k_{s}=0.9 k_{D}$.

In order to better see the behavior of the local DOS, we plot in Fig. 5(a) $\tilde{\rho}(\mathbf{k}, \varepsilon)$ of the graphene overlayer as a function of $\varepsilon$ at $11 \mathbf{k}$ points on the $k_{x}$ axis in the neighborhood of $\mathbf{k}_{D}$. The corresponding $y$ and $z$ components of the magnetization density of the graphene overlayer are shown in Fig. 5(b). In both panels, $\Delta \varepsilon=20 \mathrm{meV}$ and $\gamma=2 \mathrm{meV}$. The linear dispersion of the $\pi$ and $\pi^{*}$ bands is also visible in these DOS curves. Furthermore, we see that the DOS peaks of both bands become narrower as $\mathbf{k}$ moves away from the $K$ point of graphene. As an example, let us look at the DOS peaks of the $\pi$ and $\pi^{*}$ bands at $k_{x}=0.925 k_{D}$ marked by arrows 1 and $1^{\prime}$ in Figs. 4 and 5. It is seen in Fig. 2(a) that at $k_{x}=0.925 k_{D}$ $\left(1.03 k_{s}\right)$, the energies of the two peaks 1 and $1^{\prime}$ are located within a projected bulk band gap of $\operatorname{Ir}(111)$. Nevertheless, due to the backfolding of the projected bulk Ir bands induced by the supercell potential, both the bands exhibit a nonvanishing energy width. More importantly, the corresponding $\tilde{s}_{y}(\mathbf{k}, \varepsilon)$ and $\tilde{s}_{z}(\mathbf{k}, \varepsilon)$ in Fig. 5(b) reveals that the $\pi$ and $\pi^{*}$ bands exhibit a spin splitting due to orbital hybridizations with Ir $5 d$ states. We made a supplemental Green's function calculation 
with a finer energy interval $\Delta \varepsilon=2 \mathrm{meV}$ and $\gamma=1 \mathrm{meV}$ in the energy range of the $\pi$ and $\pi^{*}$ bands and obtained the local DOS shown in the two insets of Fig. 5(a). It is seen that the lower and upper DOS peaks of the spin-split $\pi$ band have a FWHM of $\sim 10$ and $\sim 20 \mathrm{meV}$, respectively, and the energy splitting between them is $30 \mathrm{meV}$ as shown in the inset (i) of Fig. 5(a). This value is in rough agreement with the ARPES measurement of Marchenko et al., who reported a spin-orbit splitting of $(48 \pm 5) \mathrm{meV}$ for the $\pi$ band peak at $-0.6 \mathrm{eV}$ below $E_{F}$. The magnitude of the spin-orbit splitting of the $\pi$ band in the present calculation is also in good agreement with a recent slab calculation [29]. Regarding the $\pi^{*}$ band, while its lower DOS peak is much broader than the upper one as shown in the inset (ii) of Fig. 5(a), the energy splitting between the two peaks, $\sim 30 \mathrm{meV}$, is similar to that of the $\pi$ band.

Figure 5(b) shows that the spin texture in the $\pi$ and $\pi^{*}$ bands is qualitatively different from that in typical surface bands exhibiting a large Rashba spin splitting; for instance, the $L$-gap surface band on $\mathrm{Au}(111)$ [42]. The normal (z) component is about the same size as the parallel $(y)$ one. Moreover, the parallel $(y)$ component nearly vanishes at the $K$ point due to the approximate $C_{3 v}$ symmetry of the overlayer. In this relation, we refer to a recent paper of Sławińska and Cerdá [45], in which the complex spin texture in the graphene $\pi$ bands on $\mathrm{Pt}(111)$ and $\mathrm{Ni}(111) / \mathrm{Au}$ was investigated in detail by a large-scale DFT calculation.

The DOS in the region surrounded by oval A in Fig. 4 reveals that the $\pi$ band interacts strongly with the $S_{1}$ band of $\operatorname{Ir}(111)$, leading to the opening of a gap and strong hybridizations of the two components. The same gap opening happened in the $(1 \times 1)$ overlayer presented in Sec. III B and is in line with the experiment reporting a strong distortion of the linear dispersion curve of the $\pi$ band near $E_{F}$ [18]. The energy gap formed between the $\pi$ and $S_{1}$ bands can also be seen in Fig. 5(a) as a dip in the DOS curves at $k_{x}=0.95$ and $0.975 k_{D}$ (see ovals labeled A). Similarly, the DOS in the region surrounded by oval E in Fig. 4 suggests that the $\pi$ band strongly hybridizes with the $S_{2}$ band of $\operatorname{Ir}(111)$ as in the case of the $(1 \times 1)$ overlayers shown in Fig. 3 .

Because of the strong modification of the dispersion curves caused by the interaction between the $\pi$ and $S_{1}$ band, the actual Dirac point $E_{D}$, at which the upper and lower Dirac cones cross, does not coincide with the one estimated by extrapolating the occupied part of linear dispersion of the $\pi$ band. As exemplified by a dashed yellow line in Fig. 4(b), the Dirac point estimated from linear dispersion of the $\pi$ band may be only slightly above $E_{F}$, which is in agreement with previous experiments. However, in fact, as marked by arrow 2 in Fig. 4(a), the $\pi$ and $\pi^{*}$ bands at $k_{x}=k_{D}$ form a single broad peak at $0.43 \mathrm{eV}$ above $E_{F}$. It is seen from Fig. 5(a) that its FWHM amounts to $\sim 0.22 \mathrm{eV}$, which is about ten times larger than the corresponding one for the $(1 \times 1)$ overlayer shown in Fig. 3(a). An intuitive explanation of why no appreciable gap emerges between the upper and lower bands is as follows: As mentioned in Sec. III B, $E_{g}$ appears when the carbon atoms in the $C_{1}$ and $C_{2}$ sites become symmetrically inequivalent. In the present case, within the $(10 \times 10)$ unit cell, the $C_{1}$ sites are partly on atop, hcp, and fcc sites as illustrated in Fig. 1, and importantly, this is true for the $C_{2}$ sites. In this sense, both the sites are symmetrically equivalent when averaged over the

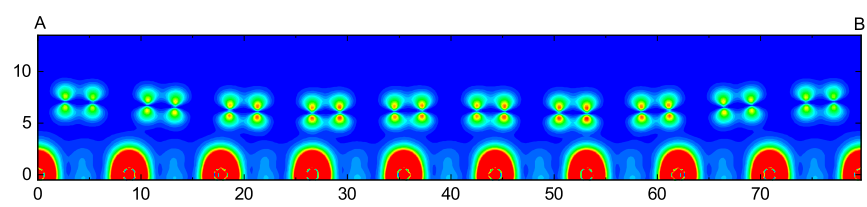

FIG. 6. Charge density in the energy range $0.32 \mathrm{eV} \leqslant \varepsilon-E_{F} \leqslant$ $0.54 \mathrm{eV}$ at the $K$ point of graphene corresponding to the FWHM of the broad DOS peak at $E_{D}$ on a vertical cut plane along the diagonal line $A B$ in Fig. 1.

supercell; as a result, the gap does not appear. The present result on the $\pi$ state at the $K$ point of graphene is not in line with the slab calculation of Dedkov and Voloshina [1], in which $E_{D}$ and $E_{g}$ were estimated to be $150 \mathrm{meV}$ above $E_{F}$ and $\sim 300 \mathrm{meV}$, respectively. We believe that in general, it may be difficult to predict the formation of a broad resonant peak as shown in Fig. 5 by a slab calculation since the resonance is replaced by a finite number of discrete energy levels.

The present result suggests that the strength of the overlayer-substrate interaction and the local symmetry of a small portion of the overlayer are not sufficient to determine the gap opening at $E_{D}$. Rather, the overall symmetry of the overlayer in a whole unit cell plays a crucial role in the opening of a gap. Restructuring or symmetry lowering of the overlayer via the adsorption and intercalation of atoms results in the opening of an energy gap. In fact, the intercalation of $\mathrm{Cu}$ [23] and $\mathrm{Bi}$ [24] in between the graphene and the $\operatorname{Ir}(111)$ surface was found to induce the lowering of $E_{D}$ below $E_{F}$ and the opening of an energy gap $E_{g}$, whereas no clear evidence of the gap opening was observed for the $\mathrm{Gd}$ intercalation [25]. In the case of the $\mathrm{Cu}$ intercalation, it was shown that the hybridization of the graphene $\pi$ and $\mathrm{Cu} 3 d$ states is responsible for the gap opening at $E_{D}$ [23].

In Fig. 6 we show the electron charge density in the energy range $0.32 \mathrm{eV} \leqslant \varepsilon-E_{F} \leqslant 0.54 \mathrm{eV}$ corresponding to the FWHM of the broad $\pi$ band peak on a vertical cut plane along the diagonal line $A B$ in Fig. 1. As is seen, the charge density is delocalized over the whole $(10 \times 10)$ unit cell although its amplitude on the atop site is a little smaller than those at the hcp and fcc sites. It is also seen that the electronic states in this energy range are strongly hybridized with the Ir states.

In Fig. 4, we observe several replica Dirac cones. As illustrated in Fig. 7, these Dirac cones are centered at $\mathbf{k}_{D}+\mathbf{G}_{i}$. First, within the interval $0.8 \leqslant k_{x} / k_{D} \leqslant 0.9\left(1.1 \leqslant k_{x} / k_{D} \leqslant\right.$ 1.2), we observe two replica $\pi$ bands centered at $\mathbf{k}_{\mathbf{D}}+\mathbf{G}_{1}$ and $\mathbf{k}_{\mathbf{D}}+\mathbf{G}_{2}\left(\mathbf{k}_{\mathbf{D}}+\mathbf{G}_{3}\right.$ and $\left.\mathbf{k}_{\mathbf{D}}+\mathbf{G}_{4}\right)$ as marked by two arrows 3 and 4 (5 and 6). The two bands interact with each other within the interval and split into two bands dispersing parallel to each other with $k_{x}$. In contrast, the replica $\pi^{*}$ bands marked by arrow $3^{\prime}\left(5^{\prime}\right)$ do not exhibit a significant energy splitting within the same interval $0.8 \leqslant k_{x} / k_{D} \leqslant 0.9\left(1.1 \leqslant k_{x} / k_{D} \leqslant\right.$ 1.2 ), implying that the interaction between the two replica $\pi^{*}$ bands is much weaker. In addition, in Fig. 4(b), we see replica $\pi$ and $\pi^{*}$ bands centered at $\mathbf{k}_{\mathbf{D}}+\mathbf{G}_{5}$ as marked by arrows 7 and $7^{\prime}$. Also, in Fig. 4(a), we observe a weak DOS signal of replica Dirac cones centered at $\mathbf{k}_{\mathbf{D}}+\mathbf{G}_{7}$ as marked by two arrows 8 and $8^{\prime}$. 


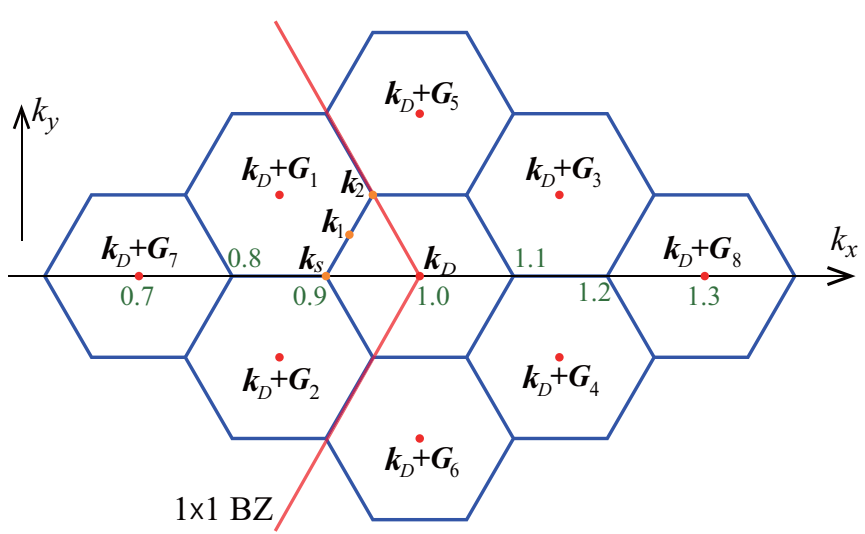

FIG. 7. Red line indicates a small portion of the first BZ of the $(1 \times 1)$ graphene near $\mathbf{k}_{D}=\left(k_{D}, 0\right)$, the $K$ point on the $k_{x}$ axis. Small hexagons are mini BZs of the $(10 \times 10)$ supercell whose centers are chosen to be at $\mathbf{k}_{D}+\mathbf{G}_{i}$ where $\mathbf{G}_{i}$ is a reciprocal lattice vector of the supercell. Replica Dirac cones centered at neighboring $\mathbf{k}_{D}+\mathbf{G}_{i}$ 's interact on the boundaries of the mini BZs, leading to the opening of a mini energy gap. Small numbers along the $k_{x}$ axis indicate $k_{x} / k_{D}$.

In the past, mini gaps at the crossing of the primary $\pi$ band and its replicas were investigated extensively by ARPES [15-20]. As indicated by the two ovals B and C in Fig. 4(a), we observe a mini gap at $\mathbf{k}=\left(0.9 k_{D}, 0\right)$ and $\left(1.1 k_{D}, 0\right)$ where the primary $\pi$ band crosses with two of its replicas. Also, as indicated by oval D in Fig. 4(b) a mini gap appears at $\mathbf{k}=\left(k_{D}, \sqrt{3} k_{D} / 20\right)$ where the primary $\pi$ band crosses with its replica centered at $\mathbf{k}+\mathbf{G}_{5}$. The mini gaps $\mathrm{B}$ and $\mathrm{C}$ are more clearly visible in the DOS curves at $k_{x}=0.9 k_{D}$ and $1.1 k_{D}$ in Fig. 5 (see ovals B and C).

In order to elucidate more details of the local DOS in the energy range of mini gaps, we performed a supplemental Green's function calculation with $\Delta \varepsilon=2 \mathrm{meV}$ and $\gamma=$ $1 \mathrm{meV}$ at $\mathbf{k}=\mathbf{k}_{s}, \mathbf{k}_{1}$, and $\mathbf{k}_{2}$ on the boundary line of the mini BZ shown in Fig. 7. The calculated $\tilde{\rho}(\mathbf{k}, \varepsilon)$ and $\tilde{s}_{z}(\mathbf{k}, \varepsilon)$ at these points are shown in Fig. 8. By virtue of the smaller energy spacing $\Delta \varepsilon$ we can resolve more DOS peaks than those in Fig. 5. In Figs. 8(a) and 8(b), we observe six DOS peaks since three Dirac cones intersect at $\mathbf{k}=\mathbf{k}_{s}$ and each of them possesses two spin components. One should observe also six states at $\mathbf{k}=\mathbf{k}_{2}$. Indeed, although there appear in Figs. 8(e) and 8(f) only five peaks, the lowest peak at $-0.66 \mathrm{eV}$ below $E_{F}$, which possesses a larger area than the two neighboring ones at higher energies, comprises two nearly degenerate states. In Figs. 8(c) and 8(d), we see two spin-split $\pi$ band peaks at the lower and upper sides of the mini gap since only two Dirac cones cross at $\mathbf{k}=\mathbf{k}_{1}$.

The gap size, if defined as the maximum of the energy differences between two nearest-neighbor DOS peaks in Fig. 8, amount to 310,138 , and $88 \mathrm{meV}$ at $\mathbf{k}=\mathbf{k}_{s}, \mathbf{k}_{1}$, and $\mathbf{k}_{2}$, respectively. Alternatively, since the fine DOS structures comprising more than one peak have not been experimentally observed so far, it might be better for comparison with experiments to compute the centers of gravity of the DOS on the lower and upper sides of the energy gap separately and to define the gap size as the energy difference between the two values. The gap sizes estimated in this way are $\sim 340, \sim 170$, and $\sim 130 \mathrm{meV}$
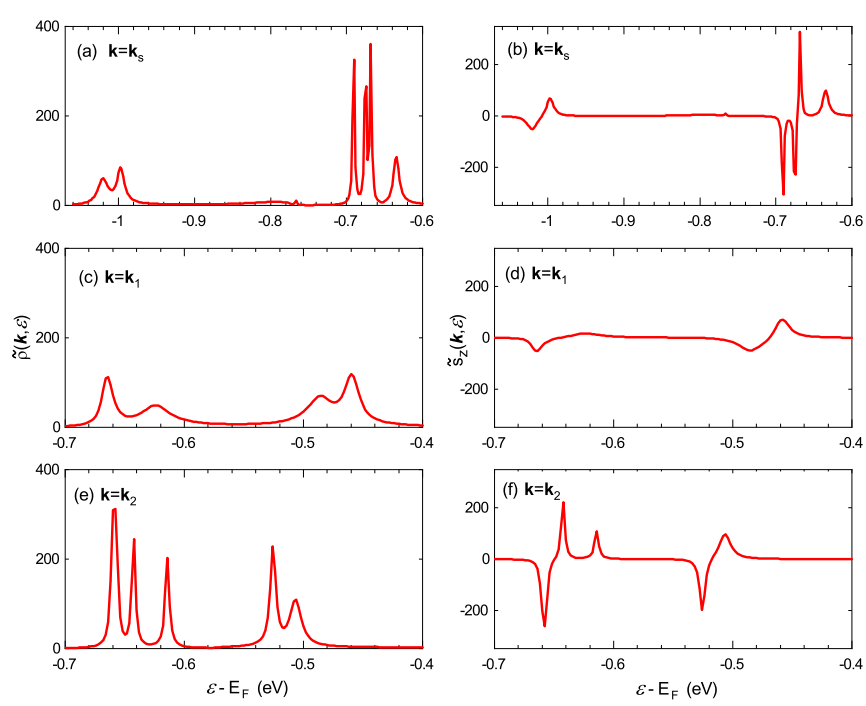

FIG. 8. $\tilde{\rho}(\mathbf{k}, \varepsilon)$ at (a) $\mathbf{k}=\mathbf{k}_{s}$, (c) $\mathbf{k}=\mathbf{k}_{1}$, and (e) $\mathbf{k}=\mathbf{k}_{2} \cdot \tilde{s}_{z}(\mathbf{k}, \varepsilon)$ at (b) $\mathbf{k}=\mathbf{k}_{s}$, (d) $\mathbf{k}=\mathbf{k}_{1}$, and (f) $\mathbf{k}=\mathbf{k}_{2}$. In all panels, $\Delta \varepsilon=2 \mathrm{meV}$ and $\gamma=1 \mathrm{meV}$.

at $\mathbf{k}=\mathbf{k}_{s}, \mathbf{k}_{1}$, and $\mathbf{k}_{2}$, respectively. The gap size measured by ARPES along the line between $\mathbf{k}_{s}$ and $\mathbf{k}_{2}$ was nearly constant and amounted to $\sim 160 \mathrm{meV}$ [18] and $\sim 170 \mathrm{meV}$ [20]. Thus, the present calculation significantly overestimates the gap size at $\mathbf{k}=\mathbf{k}_{s}$. On the other hand, the present result agrees with the previous experiments $[18,20]$ in that the energy gap is located at the lowest position relative to $E_{F}$ at $\mathbf{k}_{s}$ and shifts to higher energies as $\mathbf{k}$ moves toward $\mathbf{k}_{2}$. We also note that the theoretically predicted gap closing at $\mathbf{k}=\mathbf{k}_{1}$ [46] does not occur in the present calculation, which indicates that treating the substrate as a weak perturbation potential is not accurate enough to describe the present system where the graphene $\pi$ states are strongly admixed with the substrate Ir states.

\section{CONCLUSION}

We have studied the electronic structure of a graphene monolayer on a semi-infinite $\operatorname{Ir}(111)$ surface by a firstprinciples DFT calculation. To do so, we employed a realistic structure model in which the $(10 \times 10)$ unit cell of graphene matches a $(9 \times 9)$ cell of the $\operatorname{Ir}(111)$ substrate. The surface geometry was optimized within the LDA in DFT by using a slab model containing a graphene monolayer and four $\operatorname{Ir}(111)$ atomic layers. The surface geometry obtained was used as input structural data for the subsequent EGF calculation. By exploiting the advantage of a semi-infinite surface calculation, we have clarified many more details of the energy dispersion of the graphene $\pi$ and $\pi^{*}$ bands than could be studied with standard slab calculations as follows.

We found that overall, the linear dispersion of the $\pi$ and $\pi^{*}$ bands is preserved. Nevertheless, it has been revealed that their dispersion curves are significantly modified from those of freestanding graphene in several points: (i) Both the bands exhibit a Rashba-type spin splitting of $\sim 30 \mathrm{meV}$. (ii) Mini gaps appear at the crossing of the primary $\pi$ band and its replicas and their size varies between $\sim 100$ and $\sim 300 \mathrm{meV}$. (iii) The energy dispersion of the $\pi$ band in the vicinity of $E_{F}$ 
is strongly modified due to the gap opening at the crossing of the $\pi$ band and the $S_{1}$ band of $\operatorname{Ir}(111)$. (iv) The $\pi$ and $\pi^{*}$ bands form a heavily broadened single peak at the $K$ point of graphene and its peak position $E_{D}$ is located at a higher energy than expected from the linear dispersion of the occupied part of the $\pi$ band. (v) No appreciable gap $E_{g}$ appears at $E_{D}$ since the two carbon cites in a primitive unit cell of graphene become virtually equivalent when averaged over the $(10 \times 10)$ supercell. (vi) The energy widths of the $\pi$ and $\pi^{*}$ bands become progressively narrower as $\mathbf{k}$ moves away from the $K$ point.

\section{ACKNOWLEDGMENT}

This work is supported by the Japan Society for the Promotion of Science (JSPS) KAKENHI, Grants No. JP18H01875 and No. JP20K05333.

\section{APPENDIX: UNFOLDED LOCAL DENSITY OF STATES}

In this Appendix we describe a band-unfolding method suitable for the surface Green's function formalism. Let us assume that a crystal surface, which has a primitive lattice (PL) structure specified by two basic lattice vectors $\left\{\mathbf{a}_{1}, \mathbf{a}_{2}\right\}$, undergoes a structural change to result in a relaxed structure described by a commensurate superlattice (SL) specified by two basic lattice vectors, $\mathbf{A}_{1}=m_{11} \mathbf{a}_{1}+m_{12} \mathbf{a}_{2}$ and $\mathbf{A}_{2}=$ $m_{21} \mathbf{a}_{1}+m_{22} \mathbf{a}_{2}$. For example, $m_{11}=m_{12}=m_{21}=m_{22}=10$ in the case of the $(10 \times 10)$ graphene overlayer on $\operatorname{Ir}(111)$. We use a lowercase $\mathbf{g}$ and an uppercase $\mathbf{G}$ to represent reciprocal lattice vectors of the PL and SL, respectively. The 2D unit cell of the SL is $N=\left|m_{11} m_{22}-m_{12} m_{21}\right|$ times larger than that of the PL, while the BZ of the PL is $N$ times larger than that of the SL. Given a $2 \mathrm{D}$ wave vector $\mathbf{k}$ in the first $\mathrm{BZ}$ of the SL, one can find $N$ reciprocal lattice vectors $\mathbf{G}_{i}(i=1,2, \ldots, N)$, for which $\mathbf{k}_{i}=\mathbf{k}+\mathbf{G}_{i}$ lies within the first BZ of the PL. The band unfolding in the present context means to derive the local DOS of a surface atom at $N \mathbf{k}_{i}$ points in the first BZ of the PL from the surface Green's function calculated at $\mathbf{k}$ in the $\mathrm{BZ}$ of the SL.

We define a unit cell of the SL by $S=\bigcup_{j=1}^{N} s_{j}$, where $s_{j}=\left\{\mathbf{l}_{j}+x_{1} \mathbf{a}_{1}+x_{2} \mathbf{a}_{2} \mid 0 \leqslant x_{1}, x_{2} \leqslant 1\right\}$ is the unit cell of the PL specified by $\mathbf{l}_{j}$, a lattice vector of the PL. We assume that all atoms in the original PL persist during the structural change and the spatial coordinate of the $n$th atom belonging to $s_{j}$ after the structural change is denoted by $\mathbf{r}_{n}\left(\mathbf{l}_{j}\right)\left(1 \leqslant n \leqslant N_{a}\right.$ and $1 \leqslant j \leqslant N$ ).

Now, let us consider the k-resolved DOS of the $n$th atom $\left(1 \leqslant n \leqslant N_{a}\right)$ defined by Eq. (2) averaged over the $N$ inequivalent unit cells $s_{j}(1 \leqslant j \leqslant N)$ forming $S$,

$$
\bar{\rho}(\mathbf{k}, \varepsilon)=\sum_{j=1}^{N} \frac{-1}{N \pi} \int_{\left|\mathbf{r}-\mathbf{r}_{n}\left(\mathbf{l}_{j}\right)\right| \leqslant R} d \mathbf{r} \operatorname{Tr}[\operatorname{Im} \hat{G}(\mathbf{k}, \omega, \mathbf{r}, \mathbf{r})]
$$

with $\omega=\varepsilon+i \gamma$.

In the embedded Green's function formalism, the $2 \times 2$ Green's function matrix in Eq. (A1) is expanded as

$$
\hat{G}\left(\mathbf{k}, \omega, \mathbf{r}, \mathbf{r}^{\prime}\right)=\sum_{\mathbf{G}, \mathbf{G}^{\prime}} \hat{G}\left(\mathbf{k}+\mathbf{G}, \mathbf{k}+\mathbf{G}^{\prime}, \omega, \mathbf{r}, \mathbf{r}^{\prime}\right),
$$

with

$$
\begin{aligned}
& \hat{G}\left(\mathbf{k}+\mathbf{G}, \mathbf{k}+\mathbf{G}^{\prime}, \omega, \mathbf{r}, \mathbf{r}^{\prime}\right) \\
& \quad=\sum_{n, n^{\prime}} \hat{G}\left(\mathbf{k}+\mathbf{G}, \mathbf{k}+\mathbf{G}^{\prime}, \omega, n, n^{\prime}\right) f_{\xi}(\mathbf{r}) f_{\xi^{\prime}}^{*}\left(\mathbf{r}^{\prime}\right),
\end{aligned}
$$

where $\xi=(\mathbf{k}+\mathbf{G}, n)$ is a composite index for the LAPW basis functions. In the interstitial region, the basis function is given as

$$
f_{\xi}(\mathbf{r})=\sqrt{\frac{2}{A L}} e^{i(\mathbf{k}+\mathbf{G}) \cdot \mathbf{x}} \sin \left(p_{n} z\right),
$$

where $A$ is the unit-cell area of the SL and $p_{n}=\frac{\pi}{L} n$ $(n=1,2,3, \ldots)$ with $L$, a parameter chosen slightly larger than the thickness of the embedded region. Within the muffintin sphere of an atom centered at $\mathbf{r}_{n}$,

$$
f_{\xi}(\mathbf{r})=\sum_{l, m}\left[A_{l m} u_{l}(r)+B_{l m} \dot{u}_{l}(r)\right] Y_{l m}\left(\mathbf{r}-\mathbf{r}_{n}\right),
$$

where $u_{l}(r)$ is a radial solution of the scalar-relativistic Koelling-Harmon equation at a reference energy $\varepsilon_{n, l}$ and $\dot{u}_{l}(r)$ denotes its energy derivative [41].

The reciprocal lattice vectors of the SL in the expansion of Eq. (A2) can be uniquely decomposed into the form

$$
\mathbf{G}=\mathbf{G}_{i}+\mathbf{g},
$$

where $\mathbf{G}_{i}$ is one of the $N$ reciprocal lattice vectors as defined above and $\mathbf{g}$ is a reciprocal lattice vector of the PL. Thus, Eq. (A2) can be rewritten as

$$
\hat{G}\left(\mathbf{k}, \omega, \mathbf{r}, \mathbf{r}^{\prime}\right)=\sum_{i, i^{\prime}=1}^{N} \sum_{\mathbf{g}, \mathbf{g}^{\prime}} \hat{G}\left(\mathbf{k}_{i}+\mathbf{g}, \mathbf{k}_{i^{\prime}}+\mathbf{g}^{\prime}, \omega, \mathbf{r}, \mathbf{r}^{\prime}\right) .
$$

By inserting Eq. (A7) into Eq. (A1) we obtain

$$
\bar{\rho}(\mathbf{k}, \varepsilon)=\frac{-1}{N \pi} \sum_{j=1}^{N} \sum_{i, i^{\prime}=1}^{N} \sum_{\mathbf{g}, \mathbf{g}^{\prime}} \int_{\left|\mathbf{r}-\mathbf{r}_{n}\left(\mathbf{I}_{j}\right)\right| \leqslant R} d \mathbf{r} \operatorname{Tr}\left[\operatorname{Im} \hat{G}\left(\mathbf{k}_{i}+\mathbf{g}, \mathbf{k}_{i^{\prime}}+\mathbf{g}^{\prime}, \omega, \mathbf{r}, \mathbf{r}\right)\right] .
$$

To go further, we consider a simplified case where the atomic position of the $n$th atom in the $j$ th unit cell remains the same during the structural change and is given by $\mathbf{r}_{n}\left(\mathbf{l}_{j}\right)=\mathbf{r}_{n}+\mathbf{l}_{j}$. In the case of the $(10 \times 10)$ graphene overlayer, this means that a perfect graphene monolayer lies on top of the $\operatorname{Ir}(111)$ substrate. From Eqs. (A4) and (A5) we see that the basis function satisfies

$$
f_{\xi}\left(\mathbf{r}+\mathbf{l}_{j}\right)=e^{i(\mathbf{k}+\mathbf{G}) \cdot \mathbf{l}_{j}} f_{\xi}(\mathbf{r})=e^{i\left(\mathbf{k}+\mathbf{G}_{i}+\mathbf{g}\right) \cdot \mathbf{l}_{j}} f_{\xi}(\mathbf{r})=e^{i\left(\mathbf{k}+\mathbf{G}_{i}\right) \cdot \mathbf{l}_{j}} f_{\xi}(\mathbf{r}),
$$


if $\left|\mathbf{r}-\mathbf{r}_{n}\right| \leqslant R$ (it is necessary that the radial functions $u_{l}$ and $\dot{u}_{l}$ are the same among the $N$ atoms related by $\mathbf{l}_{j}$ ). Using Eqs. (A3) and (A9), we see that Eq. (A8) reads

$$
\bar{\rho}(\mathbf{k}, \varepsilon)=\frac{-1}{N \pi} \sum_{i, i^{\prime}=1}^{N} \sum_{\mathbf{g}, \mathbf{g}^{\prime}} \int_{\left|\mathbf{r}-\mathbf{r}_{n}\right| \leqslant R} d \mathbf{r} \operatorname{Tr} \operatorname{Im}\left[\sum_{j=1}^{N} e^{i\left(\mathbf{G}_{i}-\mathbf{G}_{i^{\prime}}\right) \cdot \mathbf{l}_{j}} \hat{G}\left(\mathbf{k}_{i}+\mathbf{g}, \mathbf{k}_{i^{\prime}}+\mathbf{g}^{\prime}, \omega, \mathbf{r}, \mathbf{r}\right)\right] .
$$

Since $\sum_{j=1}^{N} e^{i\left(\mathbf{G}_{i}-\mathbf{G}_{i^{\prime}}\right) \cdot \mathbf{l}_{j}}=N \delta_{i, i^{\prime}}$, only the diagonal terms with $i=i^{\prime}$ contribute to Eq. (A10). Thus, dropping the off-diagonal terms, Eq. (A8) reads

$$
\bar{\rho}(\mathbf{k}, \varepsilon)=\sum_{i=1}^{N} \tilde{\rho}\left(\mathbf{k}_{i}, \varepsilon\right)
$$

where $\tilde{\rho}\left(\mathbf{k}_{i}, \varepsilon\right)$ is defined by

$$
\tilde{\rho}\left(\mathbf{k}_{i}, \varepsilon\right)=\frac{-1}{N \pi} \sum_{j=1}^{N} \sum_{\mathbf{g}, \mathbf{g}^{\prime}} \int_{\left|\mathbf{r}-\mathbf{r}_{n}\left(\mathbf{l}_{j}\right)\right| \leqslant R} d \mathbf{r} \operatorname{Tr}\left[\operatorname{Im} \hat{G}\left(\mathbf{k}_{i}+\mathbf{g}, \mathbf{k}_{i}+\mathbf{g}^{\prime}, \omega, \mathbf{r}, \mathbf{r}\right)\right] .
$$

Equation (A11) indicates the local DOS of the $n$th atom averaged over the $N$ unit cells $s_{j}(j=1,2, \ldots, N)$ can be decomposed into $N$ DOS functions at the unfolded $\mathbf{k}$ points, $\mathbf{k}_{i}=\mathbf{k}+\mathbf{G}_{i}$. In fact, in the case of the $(10 \times 10)$ graphene overlayer on $\operatorname{Ir}(111)$, the positions of the $\mathrm{C}$ atoms move from $\mathbf{r}_{n}\left(\mathbf{l}_{j}\right)=\mathbf{r}_{n}+\mathbf{l}_{j}$ due to surface relaxations. Hence, strictly speaking, Eq. (A11) does not hold. However, we expect that the contribution of the nondiagonal terms with $i \neq i^{\prime}$ in Eq. (A8) may be much smaller than the diagonal ones since there should be significant cancellations when the sum is taken over the $N \mathbf{l}_{j}$ vectors. Hence, we define Eq. (A12) as the unfolded local DOS of the $n$th atom at $\mathbf{k}_{i}$ in the present work. Similarly, the $\alpha$ component of the unfolded magnetization density of the $n$th atom, $\tilde{s}_{\alpha}\left(\mathbf{k}_{i}, \varepsilon\right)$, is defined by replacing $\hat{G}$ in Eq. (A12) by $\hat{\sigma}_{\alpha} \hat{G}$.

[1] Y. Dedkov and E. Voloshina, Graphene growth and properties on metal substrates, J. Phys.: Condens. Matter 27, 303002 (2015).

[2] E. Voloshina and Y. Dedkov, Graphene on metallic surfaces: Problems and perspectives, Phys. Chem. Chem. Phys. 14, 13502 (2012).

[3] E. Voloshina and Y. Dedkov, General approach to the understanding the electronic structure of graphene on metals, Mater. Res. Express 1, 035603 (2014).

[4] X. Xu, C. Liu, Z. Sun, T. Cao, Z. Zhang, E. Wang, Z. Liu, and K. Liu, Interfacial engineering in graphene bandgap, Chem. Soc. Rev. 47, 3059 (2018).

[5] C. Gong, G. Lee, B. Shan, E. M. Vogel, R. M. Wallace, and K. Cho, First-principles study of metal-graphene interfaces, J. Appl. Phys. 108, 123711 (2010).

[6] L. Zhong, J. Li, Y. Li, H. Lu, H. Du, L. Gan, C. Xu, S. W. Chiang, and F. Kang, Unraveling the influence of metal substrates on graphene nucleation from first-principles study, J. Phys. Chem. C 120, 23239 (2016).

[7] B. J. Schultz, R. V. Dennis, V. Lee, and S. Banerjee, An electronic structure perspective of graphene interfaces, Nanoscale 6, 3444 (2014).

[8] N. M. R. Peres, F. Guinea, and A. H. Castro Neto, Electronic properties of disordered two-dimensional carbon, Phys. Rev. B 73, 125411 (2006).

[9] A. T. N'Diaye, S. Bleikamp, P. J. Feibelman, and T. Michely, Two-Dimensional Ir Cluster Lattice on a Graphene Moiré on Ir(111), Phys. Rev. Lett. 97, 215501 (2006).

[10] A. T. N'Diaye, J. Coraux, T. N. Plasa, C. Busse, and T. Michely, Structure of epitaxial graphene on $\operatorname{Ir}(111)$, New J. Phys. 10, 043033 (2008).
[11] C. Busse, P. Lazić, R. Djemour, J. Coraux, T. Gerber, N. Atodiresei, V. Caciuc, R. Brako, A. T. N'Diaye, S. Blügel, J. Zegenhagen, and T. Michely, Graphene on $\operatorname{Ir}(111)$ : Physisorption with Chemical Modulation, Phys. Rev. Lett. 107, 036101 (2011).

[12] S. K. Hämäläinen, M. P. Boneschanscher, P. H. Jacobse, I. Swart, K. Pussi, W. Moritz, J. Lahtinen, P. Liljeroth, and J. Sainio, Structure and local variations of the graphene moiré on Ir(111), Phys. Rev. B 88, 201406(R) (2013).

[13] F. Jean, T. Zhou, N. Blanc, R. Felici, J. Coraux, and G. Renaud, Topography of the graphene/Ir(111) moiré studied by surface x-ray diffraction, Phys. Rev. B 91, 245424 (2015).

[14] K. M. Omambac, H. Hattab, C. Brand, G. Jnawali, A. T. N'Diaye, J. Coraux, R. van Gastel, B. Poelsema, T. Michely, F.-J. Meyer zu Heringdorf, and M. Horn-von Hoegen, Temperature-controlled rotational epitaxy of graphene, Nano Lett. 19, 4594 (2019).

[15] D. Marchenko, J. Sánchez-Barriga, M. R. Scholz, O. Rader, and A. Varykhalov, Spin splitting of Dirac fermions in aligned and rotated graphene on $\operatorname{Ir}(111)$, Phys. Rev. B 87, 115426 (2013).

[16] I. Pletikosić, M. Kralj, P. Pervan, R. Brako, J. Coraux, A. T. N'Diaye, C. Busse, and T. Michely, Dirac Cones and Minigaps for Graphene on $\operatorname{Ir}(111)$, Phys. Rev. Lett. 102, 056808 (2009).

[17] M. Kralj, I. Pletikosić, M. Petrović, P. Pervan, M. Milun, A. T. N'Diaye, C. Busse, T. Michely, J. Fujii, and I. Vobornik, Graphene on $\operatorname{Ir}(111)$ characterized by angle-resolved photoemission, Phys. Rev. B 84, 075427 (2011).

[18] E. Starodub, A. Bostwick, L. Moreschini, S. Nie, F. E. Gabaly, K. F. McCarty, and E. Rotenberg, In-plane orientation effects 
on the electronic structure, stability, and Raman scattering of monolayer graphene on $\operatorname{Ir}(111)$, Phys. Rev. B 83, 125428 (2011).

[19] S. Rusponi, M. Papagno, P. Moras, S. Vlaic, M. Etzkorn, P. M. Sheverdyaeva, D. Pacilé, H. Brune, and C. Carbone, Highly Anisotropic Dirac Cones in Epitaxial Graphene Modulated by an Island Superlattice, Phys. Rev. Lett. 105, 246803 (2010).

[20] J. Sánchez-Barriga, A. Varykhalov, D. Marchenko, M. R. Scholz, and O. Rader, Minigap isotropy and broken chirality in graphene with periodic corrugation enhanced by cluster superlattices, Phys. Rev. B 85, 201413(R) (2012).

[21] J. Sánchez-Barriga, G. Bihlmayer, D. Wortmann, D. Marchenko, O. Rader, and A Varykhalov, Effect of structural modulation and thickness of a graphene overlayer on the binding energy of the Rashba-type surface state of $\operatorname{Ir}(111)$, New J. Phys. 15, 115009 (2013).

[22] K. Schulte, N. A. Vinogradov, M. L. Ng, N. Mårtensson, and, A. B. Preobrajenskia, Bandgap formation in graphene on $\operatorname{Ir}(111)$ through oxidation, Appl. Surf. Sci. 267, 74 (2013).

[23] H. Vita, S. Böttcher, K. Horn, E. N. Voloshina, R. E. Ovcharenko, Th. Kampen, A. Thissen, and Y. S. Dedkov, Understanding the origin of band gap formation in graphene on metals: Graphene on Cu/Ir(111), Sci. Rep. 4, 5704 (2014).

[24] J. Warmuth, A. Bruix, M. Michiardi, T. Hänke, M. Bianchi, J. Wiebe, R. Wiesendanger, B. Hammer, P. Hofmann, and A. A. Khajetoorians, Band-gap engineering by $\mathrm{Bi}$ intercalation of graphene on $\operatorname{Ir}(111)$, Phys. Rev. B 93, 165437 (2016).

[25] I. I. Klimovskikh, M. Krivenkov, A. Varykhalov, D. Estyunin, and A. M. Shikin, Reconstructed Fermi surface in graphene on $\operatorname{Ir}(111)$ by Gd-Ir surface alloying, Carbon 147, 182 (2019).

[26] Y. Dedkov and E. Voloshina, Spectroscopic and DFT studies of graphene intercalation systems on metals, J. Electron. Spectrosc. Relat. Phenom. 219, 77 (2017).

[27] D. Pacilé, P. Leicht, M. Papagno, P. M. Sheverdyaeva, P. Moras, C. Carbone, K. Krausert, L. Zielke, M. Fonin, Y. S. Dedkov, F. Mittendorfer, and J. Doppler, Artificially lattice-mismatched graphene/metal interface: Graphene/Ni/Ir(111), Phys. Rev. B 87, 035420 (2013).

[28] E. N. Voloshina, E. Fertitta, A. Garhofer, F. Mittendorfer, M. Fonin, A. Thissen, and Y. S. Dedkov, Electronic structure and imaging contrast of graphene moiré on metals, Sci. Rep. 3, 1072 (2013).

[29] E. Voloshina and Y. Dedkov, Realistic large-scale modeling of Rashba and induced spin-orbit effects in graphene/high-Z-metal systems, Adv. Theory Simul. 1, 1800063 (2018).
[30] T. Vincent, E. Voloshina, S. Pons, S. Simon, M. Fonin, K. Wang, B. Paulus, D. Roditchev, Y. Dedkov, and S. Vlaic, Quantum well states for graphene spin-texture engineering, J. Phys. Chem. Lett. 11, 1594 (2020).

[31] P. J. Feibelman, Pinning of graphene to $\operatorname{Ir}(111)$ by flat Ir dots, Phys. Rev. B 77, 165419 (2008).

[32] R. Brako, D. Šokčević, P. Lazić, and N. Atodiresei, Graphene on the $\operatorname{Ir}(111)$ surface: From van der Waals to strong bonding, New J. Phys. 12, 113016 (2010).

[33] I. Hamada, van der Waals density functional made accurate, Phys. Rev. B 89, 121103(R) (2014).

[34] Y. Lin, Y. Li, J. T. Sadowski, W. Jin, J. I. Dadap, M. S. Hybertsen, and R. M. Osgood, Jr., Excitation and characterization of image potential state electrons on quasi-free-standing graphene, Phys. Rev. B 97, 165413 (2018).

[35] S. De Waele, K. Lejaeghere, M. Sluydts, and S. Cottenier, Error estimates for density-functional theory predictions of surface energy and work function, Phys. Rev. B 94, 235418 (2016).

[36] G. Kresse and J. Hafner, Ab initio molecular dynamics for liquid metals, Phys. Rev. B 47, 558(R) (1993).

[37] G. Kresse and J. Furthmüller, Efficiency of ab-initio total energy calculations for metals and semiconductors using a plane-wave basis set, Comput. Mater. Sci. 6, 15 (1996).

[38] P. E. Blöchl, Projector augmented-wave method, Phys. Rev. B 50, 17953 (1994).

[39] J. E. Inglesfield, A method of embedding, J. Phys. C 14, 3795 (1981).

[40] J. E. Inglesfield, The Embedding Method for Electronic Structure (IOP Publishing, Bristol, 2015).

[41] H. Ishida, Surface-embedded Green-function method: A formulation using a linearized-augmented-plane-wave basis set, Phys. Rev. B 63, 165409 (2001).

[42] H. Ishida, Rashba spin splitting of Shockley surface states on semi-infinite crystals, Phys. Rev. B 90, 235422 (2014).

[43] D. J. Singh and L. Nordstrom, Planewaves, Pseudopotentials, and the LAPW Method, 2nd ed. (Springer, New York, 2006).

[44] H. Ishida, Bulk versus surface contributions to the Rashba spin splitting of Shockley surface states, Phys. Rev. B 98, 205412 (2018).

[45] J. Sławińska and J. Cerdá, Complex spin texture of Dirac cones induced via spin-orbit proximity effect in graphene on metals, Phys. Rev. B 98, 075436 (2018).

[46] C.-H. Park, L. Yang, Y.-W. Son, M. L. Cohen, and S. G. Louie, New Generation of Massless Dirac Fermions in Graphene under External Periodic Potentials, Phys. Rev. Lett. 101, 126804 (2008). 